\title{
BOUSSINESQ-TYPE EQUATIONS WITH VARIABLE COEFFICIENTS FOR NARROW-BANDED WAVE PROPAGATION FROM ARBITRARY DEPTHS TO SHALLOW WATERS
}

\author{
Gonzalo Simarro $^{1}$, Alvaro Galan ${ }^{2}$, Alejandro Orfila ${ }^{3}$
}

\begin{abstract}
A fully nonlinear Boussinessq-type model with 7 Nwogu's $\alpha$-like coefficients is considered. The model is one-layer and low-order to simplify the numerical solvability. The coefficients of the model are here considered functions of the local water depth so as to allow an improvement of the dispersive properties for narrow banded trains in very deep waters. The proposed model is fully nonlinear in weakly dispersive conditions, so that nonlinear wave decomposition in shallower waters is well reproduced.
\end{abstract}

Keywords: Boussinesq-type equations; linear dispersion; linear shoaling; deep waters

\section{INTRODUCTION}

Water wave propagation does not depend on water depth in deep waters. For example, the wave celerity $c$ for a wave with a period $\mathrm{T}$ is $c=g \mathrm{~T} / 2 \pi$, with $g$ the gravity acceleration. Because each wave period has a different velocity, deep waters are called dispersive. Furthermore, in deep waters the wave amplitude, $a$, is usually much smaller than the water depth $h$ and the model equations are linear (Airy theory).

As water waves propagate to the shore the water depth $h$ decreases and wave propagation becomes influenced by it. In shallow waters the wave propagation is dominated by the water depth and the wave celerity is given by $\sqrt{g h}$, independently of the wave period (i.e., non dispersive). Besides, nonlinear effects become important. An important physical characteristic of shallow waters is that the horizontal velocity profile is nearly uniform in the vertical, a fact which is exploited to obtain Nonlinear Shallow Waters Equations (NSWEs): these equations are vertically integrated.

Shallow waters correspond to $k h \lesssim 0.3$, with $k=2 \pi / \lambda$ the wave number and $\lambda$ the wave length, while $k h \gtrsim 3$ corresponds to deep waters (Dean and Dalrymple, 1984). In intermediate waters $(0.3 \lesssim k h \lesssim 3$ ) nonlinear and dispersion effects coexist and neither Airy theory nor NSWEs can properly represent the physics. To overcome this problem, two main pertubation approaches are found (Dingemans, 1997). On the one hand, Stokes theory departs from the fully dispersive linear Airy theory to incorporate weakly nonlinear effects. On the other, Boussinesq-Type Equations (BTEs) depart from NSWEs and include weakly dispersive effects. We will focus on BTEs here.

Being $a_{0}, h_{0}$ and $k_{0}$ characteristic values for wave amplitude, water depth and wave number respectively, the dimensionless parameters

$$
\epsilon \equiv \frac{a_{0}}{h_{0}}, \quad \text { and } \quad \mu \equiv k_{0} h_{0},
$$

represent nonlinear and dispersive effects respectively. The NSWEs can represent fully nonlinear waves for the nondispersive case, and the original BTEs by Peregrine (1967) included all the nonlinear non dispersive terms (NSWEs) plus the weakly nonlinear and weakly dispersive terms $O\left(\epsilon^{1} \mu^{2}\right)$, but disregarded the highly nonlinear and weakly dispersive terms $O\left(\epsilon^{2} \mu^{2}, \epsilon^{3} \mu^{2}\right)$. The inclusion of the highly nonlinear and weakly dispersive terms $O\left(\epsilon^{2} \mu^{2}, \epsilon^{3} \mu^{2}\right)$ was done, e.g., by Green and Naghdi (1976) and Wei et al. (1995).

Equations by Peregrine (1967) were derived for the vertically averaged horizontal velocity and give good linear dispersive performance (errors below $1 \%$ relative to Airy's celerity) up to $k h \lesssim 1.1$. To improve the range of applicability, several different approaches are found in the literature. Two of them are higher order and multilayer models: higher order models include terms $O\left(\mu^{4}\right)$ or higher Gobbi et al. (2000), while multilayer models will split the flow into several layers, applying low order models into each one Lynett and Liu (2004). These two kind of models increase the numerical complexity for they include fifth order derivatives or more unknowns.

Using a low order one-layer model, Nwogu (1993) improved the linear dispersive performance up to $k h \lesssim 3.3$ by using the horizontal velocity $\mathbf{u}_{\alpha}$ at $z=z_{\alpha}$ instead of the depth averaged velocity proposed by Peregrine (1967). Above mentioned models by Wei et al. (1995) and Lynett and Liu (2004), amongst other, follow this idea to (further) improve the linear dispersion performance.

\footnotetext{
${ }^{1}$ Department of Marine Geology, ICM (CSIC), Spain

${ }^{2}$ Department of Civil Engineering, UCLM, Spain

${ }^{3}$ Department of Oceanography, IMEDEA (UIB-CSIC), Spain
} 
Following the track of low order monolayer BTEs, Madsen and Schaffer (1998) modified the equations by Wei et al. (1995) by introducing particular new terms which included free coefficients. While the equations remained exact up to $O\left(\mu^{2}\right)$, similar to those by Wei et al. (1995), for the proposed coefficients they obtained errors in linear dispersion below $1 \%$ for $k h \lesssim 6.2$.

All the above works consider that the free coefficients introduced are constant. Here we consider that these coefficients are functions of the water depth to improve the model properties up to deeper waters. As a counterpart, it will be required that the wave train travelling to the coast is, in deep waters, narrow banded (swells).

\section{GOVERNING EQUATIONS}

The fully nonlinear BTEs by Galan et al. (2012), hereafter G12, are

$$
\begin{aligned}
\mathrm{X}-\mathrm{X}_{*}+\boldsymbol{\nabla} \cdot\left[d_{1 \alpha} h^{2} \boldsymbol{\nabla} \mathrm{X}+\right. & \left.d_{2 \alpha} h^{3} \boldsymbol{\nabla} \mathrm{Y}\right]+ \\
& +\boldsymbol{\nabla} \cdot\left[\left(c_{1 \alpha} h-\frac{\eta}{2}\right) \eta \boldsymbol{\nabla X}+\left(c_{2 \alpha} h^{2}-\frac{\eta^{2}}{6}\right) \eta \boldsymbol{\nabla Y}\right]+ \\
+ & \left(\delta-\delta_{h}\right) \boldsymbol{\nabla} \cdot\left[h^{2} \boldsymbol{\nabla}\left(\mathrm{X}-\mathrm{X}_{*}\right)\right]+\delta_{h} \nabla^{2}\left[h^{2}\left(\mathrm{X}-\mathrm{X}_{*}\right)\right]+ \\
& +\delta_{\epsilon} \boldsymbol{\nabla} \cdot\left[h \eta \boldsymbol{\nabla}\left(\mathrm{X}-\mathrm{X}_{*}\right)\right]=0,
\end{aligned}
$$

and

$$
\begin{aligned}
\mathbf{Z}-\mathbf{Z}_{*}+c_{1 \alpha} h \boldsymbol{\nabla} \boldsymbol{\nabla} \cdot(h \mathbf{Z})+ & c_{2 \alpha} h^{2} \boldsymbol{\nabla} \boldsymbol{\nabla} \cdot \mathbf{Z}-\boldsymbol{\nabla}\left[\eta \boldsymbol{\nabla} \cdot(h \mathbf{Z})+\frac{\eta^{2}}{2} \boldsymbol{\nabla} \cdot \mathbf{Z}\right]+ \\
+\boldsymbol{\nabla} & {\left[\left(c_{1 \alpha} h-\eta\right) \mathbf{u} \cdot \boldsymbol{\nabla X}+\left(c_{2 \alpha} h^{2}-\frac{\eta^{2}}{2}\right) \mathbf{u} \cdot \boldsymbol{\nabla Y}+\frac{(\mathrm{X}+\eta \mathrm{Y})^{2}}{2}\right]+} \\
+\left(\gamma-\gamma_{h}\right) h^{2} \boldsymbol{\nabla} \boldsymbol{\nabla} \cdot\left(\mathbf{Z}-\mathbf{Z}_{*}\right)+\gamma_{h} h \boldsymbol{\nabla} \boldsymbol{\nabla} \cdot\left(h\left(\mathbf{Z}-\mathbf{Z}_{*}\right)\right)- & \quad-\gamma_{\epsilon} \boldsymbol{\nabla}\left[\eta \boldsymbol{\nabla} \cdot\left(h\left(\mathbf{Z}-\mathbf{Z}_{*}\right)\right)\right]=0,
\end{aligned}
$$

with $\eta$ the free surface elevation, $\mathbf{u}$ the horizontal velocity evaluated at $z=z_{\alpha}=\alpha h, \mathrm{Y} \equiv \boldsymbol{\nabla} \cdot \mathbf{u}$ and

$$
\begin{array}{rlrl}
\mathrm{X} & \equiv \boldsymbol{\nabla} \cdot(h \mathbf{u}), & \mathbf{Z} & \equiv \mathbf{u}_{t}, \\
\mathrm{X}_{*} \equiv-\eta_{t}-\boldsymbol{\nabla} \cdot(\eta \mathbf{u}), & \mathbf{Z}_{*} \equiv-\frac{1}{2} \boldsymbol{\nabla}(\mathbf{u} \cdot \mathbf{u})-g \boldsymbol{\nabla} \eta,
\end{array}
$$

with $g$ is the gravity acceleration. In equations (2)

$$
c_{1 \alpha} \equiv \alpha, \quad c_{2 \alpha} \equiv \frac{\alpha^{2}}{2}, \quad d_{1 \alpha} \equiv \alpha+\frac{1}{2}, \quad d_{2 \alpha} \equiv \frac{\alpha^{2}}{2}-\frac{1}{6},
$$

where $\alpha$ is a free coefficient, as well as $\delta, \gamma, \delta_{h}, \gamma_{h}, \delta_{\epsilon}$ and $\gamma_{\epsilon}$. Table 1 shows the values by Galan et al. (2012) and also the ones required to recover the equations by Madsen and Schaffer (1998) and Wei et al. (1995).

The equations (2) are obtained using an asymptotic expansion in $k h$ and are exact up to $O\left(\mu^{2}\right)$. Because no limitations have been imposed on the nonlinearity, they can represent fully nonlinear waves up to order $O\left(\mu^{2}\right)$. For $k h \rightarrow 0$ they tend to the exact shallow water equations. The weighting coefficients influence the behavior of the equations only in deeper waters. Being more specific, the linear dispersion (which is the feature we are concerned in here) is influenced by $\alpha, \delta$ and $\gamma$, coefficients $\delta_{h}$ and $\gamma_{h}$ only influence the linear shoaling and the coefficients $\delta_{\epsilon}$ and $\gamma_{\epsilon}$ affect only the nonlinear performance. All seven coefficients have been chosen so as to improve the linear and weakly nonlinear performance in deeper waters.

As shown by G12, the linear dispersion relationship embedded in the above equations (2) is

$$
\left\{\frac{c_{\mathrm{bte}}^{2}}{g h}=\left\{\frac{\omega^{2}}{g k_{\mathrm{bte}}^{2} h}=\frac{1-\left(d_{\alpha}+\gamma+\delta\right)\left(k_{\mathrm{bte}} h\right)^{2}+\left(d_{\alpha}+\delta\right) \gamma\left(k_{\mathrm{bte}} h\right)^{4}}{1-\left(c_{\alpha}+\gamma+\delta\right)\left(k_{\mathrm{bte}} h\right)^{2}+\left(c_{\alpha}+\gamma\right) \delta\left(k_{\mathrm{bte}} h\right)^{4}},\right.\right.
$$




\begin{tabular}{lrrr}
\hline \hline & W95 & M98 & G12 \\
\hline$\alpha$ & -0.53096 & -0.54122 & -0.54217 \\
$\delta$ & - & -0.03917 & -0.02409 \\
$\gamma$ & - & -0.01052 & -0.00492 \\
$\delta_{h}$ & - & -0.14453 & -0.15530 \\
$\gamma_{h}$ & - & -0.02153 & -0.07897 \\
$\delta_{\epsilon}$ & - & - & -0.36052 \\
$\gamma_{\epsilon}$ & - & - & 0.13169 \\
\hline \hline
\end{tabular}

Table 1: Constant coefficients for Wei et al. (1995) Madsen and Schaffer (1998), and Galan et al. (2012), denoted respectively as W95, M98 and G12.

where $c_{\mathrm{bte}}$ wave celetiry corresponding to these BTEs, $k_{\mathrm{bte}}$ the wave number, $\omega$ the wave angular frequency, $c_{\alpha} \equiv c_{\alpha, 1}+c_{\alpha, 2}=\alpha^{2} / 2+\alpha$ and $d_{\alpha} \equiv d_{\alpha, 1}+d_{\alpha, 2}$. The exact Airy dispersion expression is

$$
\left\{\frac{c_{\text {Airy }}^{2}}{g h}=\left\{\frac{\omega^{2}}{g k_{\text {Airy }}^{2} h}=\frac{\tanh \left(k_{\text {Airy }} h\right)}{k_{\text {Airy }} h} .\right.\right.
$$

For given values of gravity acceleration $g$, water depth $h$, angular frequency $\omega$ and coefficients $\alpha, \delta$ and $\gamma$, the values of $k_{\mathrm{bte}}$ and $k_{\text {Airy }}$ from the equations (5) and (6) are different in general, thus giving an error in the wave celerity (the linear dispersion error). Figure 1 shows the error in linear dispersion, defined as

$$
\varepsilon_{c} \equiv \frac{c_{\text {bte }}}{c_{\text {Airy }}}-1\left\{=\frac{k_{\text {Airy }}}{k_{\text {bte }}}-1\right\}
$$

as a function of the dimensionless group $\kappa \equiv \omega^{2} h / g$. This group, $\kappa$, can be used as a $k$-independent alternative to $\xi \equiv k h$ to evaluate whether deep or shallow waters hold Nwogu (1993). It has the advantage of not introducing $k$, which is different depending on whether equation (5) or (6) are used. For Airy theory $\kappa=\xi \tanh \xi$, and therefore, $\kappa \approx \xi$ for $\xi \gtrsim 3$.

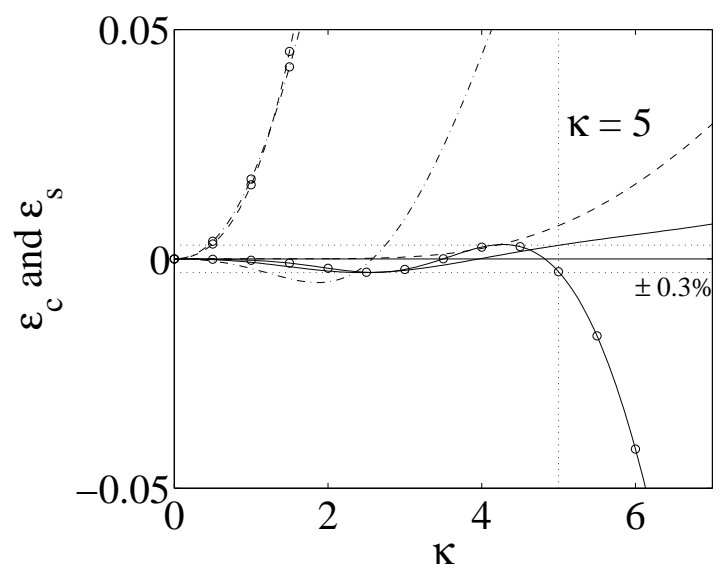

Figure 1: Errors $\varepsilon_{c}$ and $\varepsilon_{s}$ for G12 (full lines), M98 (dashed) and W95 (dash-dotted). Shoaling errors, $\varepsilon_{s}$, are denoted with symbols.

Although it is not the focus of this work, the Figure 1 also includes the error in the representation of wave amplitude assuming mild slope conditions: the relative error in the wave amplitude for a linear propagation over mild slopes from $\kappa$ to the shore Chen and Liu (1995); Lee et al. (2003); Galan et al. (2012). From Figure 1, the coefficients proposed by Galan et al. (2012) provide a better performance compared to the other sets both in linear dispersion $\left(\varepsilon_{c}\right)$ and, specially, in linear shoaling $\left(\varepsilon_{s}\right)$. 
From the figure, in deep waters the error increases. This is a natural consequence of the perturbative nature of the BTEs. By construction, using constant coefficients in the BTEs, from equation (5) one gets $c \propto \sqrt{g h}$ as $k h$ increases (deep waters), so that one could never obtain the desired result $c=g / \omega$ provided by the Airy theory in deep waters. To circumvent this problem, we will consider here that the coefficients are functions of $h$.

\section{COEFFICIENTS FUNCTIONS OF WATER DEPTH}

Let us consider that the coefficients are functions of the water depth $h$. Thinking in a dimensional way, the coefficients $\alpha, \delta$ and $\gamma$ (we focus on linear dispersion) will be functions of gravity $g$, local water depth $h$ and the limits of the angular frequencies in deep waters, $\omega_{\min }$ and $\omega_{\max }$. Applying dimensional analysis, e.g., for $\alpha$, we get

$$
\alpha=f\left(g, h, \omega_{\min }, \omega_{\max }\right)=f\left(\kappa_{\max } \equiv \frac{\omega_{\max }^{2} h}{g}, \varrho \equiv \frac{\omega_{\min }}{\omega_{\max }}\right),
$$

where $f$ stand for "function of".

In the following approach the $\omega_{\min }$ and $\omega_{\max }$ are replaced by a single frequency $\omega_{0}$, and therefore

$$
\alpha=f\left(g, h, \omega_{0}\right)=f\left(\kappa_{0} \equiv \frac{\omega_{0}^{2} h}{g}\right) .
$$

Let us consider first the deep-water propagation of monochromatic waves with an angular frequency $\omega=\omega_{0}$. In deep waters nonlinear effects are negligible and, hence, monochromatic waves remain monochromatic. In fact, the main feature to be captured by any model equations are wave celerity and amplitude.

Equation (5), which is valid for variable coefficients, and equation (6) can be understood as $k_{\text {bte }}=$ $f_{\text {bte }}(\alpha, \delta, \gamma, g, h, \omega)$ and $k_{\text {Airy }}=f_{\text {Airy }}(g, h, \omega)$. Therefore, imposing the linear dispersion to be exact, i.e., $c_{\mathrm{bte}}=c_{\text {Airy }}$, which is equivalent to impose $k_{\mathrm{bte}}=k_{\text {Airy }}$, gives the condition

$$
\left\{f_{c} \equiv\right\} f_{\text {bte }}\left(\alpha, \delta, \gamma, g, h, \omega=\omega_{0}\right)-f_{\text {Airy }}\left(g, h, \omega=\omega_{0}\right)=0 .
$$

For given values of $g, h$ and $\omega_{0}$, the above condition can be satisfied in an infinite number of ways since we have three free coefficients. However, considering, e.g., $\delta=\gamma=0$ we can obtain $\alpha$ (or $c_{\alpha}$ ) biunivocally. Recalling that $d_{\alpha}=c_{\alpha}+1 / 3$, we get

$$
c_{\alpha}=\frac{k_{0} h-\left(k_{0} h\right)^{3} / 3-\tanh \left(k_{0} h\right)}{\left(k_{0} h\right)^{2}\left(k_{0} h-\tanh \left(k_{0} h\right)\right)},
$$

where $k_{0} h$ is obtained from $\kappa_{0} \equiv \omega_{0}^{2} h / g$ since $\kappa_{0}=k_{0} h \tanh \left(k_{0} h\right)$. From $c_{\alpha}$ we recover $\alpha$ as $\alpha=-1+$ $\sqrt{1+2 c_{\alpha}}$.

The above condition (9) was already obtained by Lee et al. (2003) departing from BTEs with only one free parameter ( $\alpha$, since $\delta=\gamma=0$ are not present in their approach). Taking advantage of the fact that we have three free coefficients for linear dispersion $(\alpha, \delta$ and $\gamma)$ we will now improve the dispersion performance in a neighbourhood of $\omega=\omega_{0}$. Instead of imposing $f_{c}=0$, in order to improve the performance around $\omega_{0}$ (and to increase the number of equations up to the number of unknowns, three) we consider

$$
f_{c}\left(\omega=\omega_{0}\right)=\frac{d f_{c}}{d \omega}\left(\omega=\omega_{0}\right)=\frac{d^{2} f_{c}}{d \omega^{2}}\left(\omega=\omega_{0}\right)=0 .
$$

The above is a system of three equations for our three unknowns $c_{\alpha}$ (i.e., $\left.\alpha\right), \delta$ and $\gamma$. The analytical solutions of the above equations are shown in the appendix. There are four different sets of solutions. The first solution, " $+\&+"$ in the appendix, has values similar to those in Table 1 for M98 and G12. In all cases, the functions $\alpha, \delta$ and $\gamma$ turn out to be functions of the dimensionless group $\kappa_{0} \equiv \omega_{0}^{2} h / g$ : this fact has been anticipated through dimensional analysis.

The consequences of imposing the conditions (10) are illustrated in Figure 2 for $\omega_{0}=1 \mathrm{~s}^{-1}$ considering four different water depths $h$. The values of $\alpha, \delta$ and $\gamma$ are different at each water depth $h$ since $\kappa_{0}=\omega_{0}^{2} h / g$ changes. The error $\varepsilon_{c}$ always cancels at $\omega=\omega_{0}$ and, since the first and second derivatives are null, the error is kept small around $\omega_{0}$. In fact, for $h=250 \mathrm{~m}, 500 \mathrm{~m}$ and $1000 \mathrm{~m}$ the errors behave similarly and are 


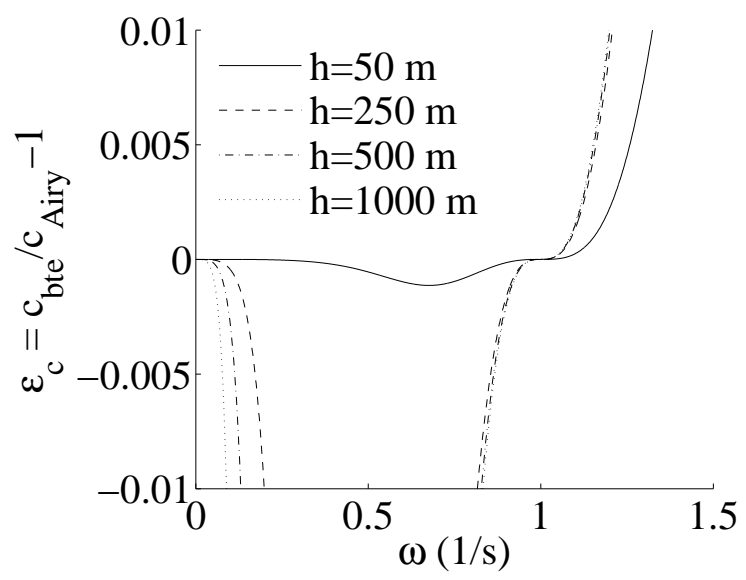

Figure 2: Illustration of the consequences of imposing $f_{c}=\partial f_{c} / \partial \omega=\partial^{2} f_{c} / \partial \omega^{2}=0$ at $\omega=\omega_{0}=1 \mathrm{~s}^{-1}$.

below $1 \%$ for $0.83 \mathrm{~s}^{-1} \leqslant \omega \leqslant 1.20 \mathrm{~s}^{-1}$. For $h=50 \mathrm{~m}$, i.e., in shallower waters, the error behaves, naturally, better: in this case the error is below $1 \%$ for $0 \leqslant \omega \leqslant 1.32 \mathrm{~s}^{-1}$. The solution " $+\&+$ " in is considered to build Figure 2.

For a given $\omega_{0}$, Figure 3 shows the range frequencies $\omega$ that can be propagated with some given errors (5\%, $1 \%$ and $0.1 \%$ ) as a function of $h$ using variable coefficients $\alpha, \delta$ and $\gamma$ obtained above. The results are presented showing the ranges $\omega / \omega_{0}$ as a function of $\kappa_{0} \equiv \omega_{0}^{2} h / g$. We recognize the very convenient fact that the curves tend to be horizontal as $h \rightarrow \infty$, so that the same range of frequencies can be propagated up to arbitrary deep waters. From Figure 3, using the coefficients as functions of $\kappa_{0}$, shown in , one can propagate in arbitrary deep waters waves the range $0.71 \omega_{0} \leqslant \omega \leqslant 1.39 \omega_{0}$ with error $\varepsilon_{c}<5 \%$, the range $0.83 \omega_{0} \leqslant \omega \leqslant 1.20 \omega_{0}$ with $\varepsilon_{c}<1 \%$ (as already stated), and the range $0.92 \omega_{0} \leqslant \omega \leqslant 1.09 \kappa_{0}$ with $\varepsilon_{c}<0.1 \%$.

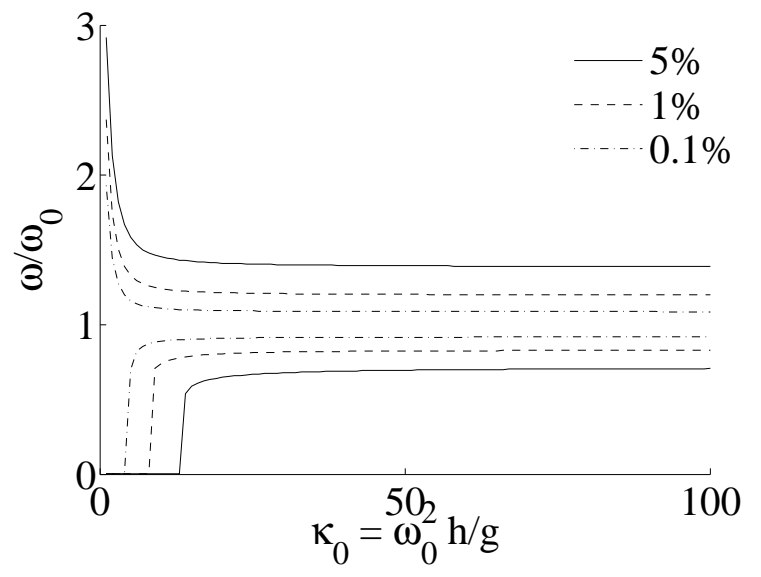

Figure 3: Range of application for the coefficients correspondig to a given $\kappa=\kappa_{0}$. The errors are $\varepsilon_{c}$.

For a given range of frequencies $\left[\omega_{\min }, \omega_{\max }\right]$ and a given maximum depth $h$, the value of $\omega_{0}$ that minimizes the error in the range, which is not necessarily the mean value $\left(\omega_{\min }+\omega_{\max }\right) / 2$, can be found. As already mentioned, in shallow waters, as it corresponds to BTEs, all frequencies are well represented. This fact is clear from Figure 3: the range $\omega / \omega_{0}$ increases as $\kappa_{0} \rightarrow 0$. For instance, for $\kappa_{0}=3$ the errors are below only $0.1 \%$ for any $\omega \lesssim 1.27 \omega_{0}$, what is to say for any $\kappa=\omega^{2} h / g \lesssim 1.27^{2} \omega_{0}^{2} h / g=1.61 \kappa_{0} \approx 4.83$. 


\section{CONCLUDING REMARKS}

The possibility of using variable coefficients (functions of the water depth) in enhanced Boussinesqtype equations has been studied and presented to improve linear dispersion in deep waters. An analytical approach is presented to obtain the variable coefficients that improve the linear dispersion performance.

\section{ACKNOWLEDGEMENT}

Authors would like to thank support from MICINN through CGL2011-22964. G. Simarro is supported by the Spanish government through the "Ramón y Cajal" program.

\section{References}

Y. Chen and P. L.-F. Liu. Modified boussinesq equations and associated parabolic models for waterwave propagation. Journal of Fluid Mechanics, 288:351-381, 1995.

R. G. Dean and R. A. Dalrymple. Water wave mechanics for engineers and scientists. Prentice-Hall, Inc., Englewoods Cliffs, New Jersey 07632, 1984.

M. W. Dingemans. Water wave propagation over uneven bottoms. World Scientific, Singapore, 1997.

A. Galan, G. Simarro, A. Orfila, J. P. Simarro, and P. L.-F. Liu. A fully nonlinear model for water wave propagation from deep to shallow waters. Journal of Waterway, Port, Coast and Ocean Engineering, page in press, 2012.

M. F. Gobbi, J. T. Kirby, and G. Wei. A fully nonlinear boussinesq model for surface waves: part ii. extension to $o(k h)^{4}$. Journal of Fluid Mechanics, 405:182-210, 2000.

A. E. Green and P. M. Naghdi. A derivation of equations for wave propagation in water of variable depth. Journal of Fluid Mechanics, 78:237-246, 1976.

C. Lee, Y.-S. Cho, and S. B. Yoon. A note on linear dispersion and shoaling properties in extended boussinesq equations. Ocean Engineering, 30:1849-1867, 2003.

P. Lynett and P. L.-F. Liu. A two layer approach to wave modeling. The Royal Society London, A, 460: 2637-2669, 2004.

P. A. Madsen and H. A. Schaffer. Higher-order boussinesq-type equations for surface gravity waves: derivation and analysis. Phil. Trans. Royal Society of London A, 356:3123-3184, 1998.

O. Nwogu. Alternative form of boussinesq equations for nearshore wave propagation. Journal of Waterway, Port, Coastal and Ocean Engineering, 119(6):618-638, 1993.

D. H. Peregrine. Long waves on a beach. Journal of Fluid Mechanics, 27:815-827, 1967.

G. Wei, J. T. Kirby, S. T. Grilli, and R. Subramnya. A fully nonlinear boussinesq model for surface waves 1. highly nonlinear unsteady waves. Journal of Fluid Mechanics, 294:71-92, 1995.

\section{APPENDIX: ANALYTICAL SOLUTIONS}

For given $g, h$ and $\omega_{0}$, the solution of equations (10) is

$$
\begin{aligned}
& \delta=\left(\varrho_{1} \pm \sqrt{\varrho_{1}^{2}-4 \varrho_{2}}\right) / 2, \\
& \gamma=\left(\varrho_{1}+1 / 3 \pm \sqrt{\varrho_{1}^{2}+1 / 9+2 \varrho_{1} / 3-4 \varrho_{3}}\right) / 2,
\end{aligned}
$$

and $c_{\alpha}=\varrho_{1}-\gamma-\delta$ so that, since $c_{\alpha} \equiv \alpha^{2} / 2+\alpha$, we can recover the coefficient $\alpha$ as $\alpha=-1+\sqrt{1+2 c_{\alpha}}$. Above

$$
\varrho_{1}=\frac{n_{1}}{3 \xi_{0}^{2} d}, \quad \varrho_{2}=\frac{n_{2}}{3 \xi_{0}^{4} d}, \quad \varrho_{3}=\frac{n_{3}}{3 \xi_{0}^{5} d},
$$


with $\xi_{0}$ verifying $\xi_{0} \tanh \xi_{0}=\kappa_{0}\left\{\equiv \omega_{0}^{2} h / g\right\}$ and

$$
\begin{aligned}
n_{1} & \equiv 6\left\{2 s_{0}^{2} \xi_{0}^{2}+5\right\} t_{0}^{2}+\left\{2 s_{0}^{2} \xi_{0}^{4}+\left(-12 s_{0}^{2}+1\right) \xi_{0}^{2}-6\left(7 s_{0}^{2}+3\right)\right\} \xi_{0} t_{0}+ \\
& +\left\{-s_{0}^{2} \xi_{0}^{2}+6\left(2 s_{0}^{4}+3 s_{0}^{2}\right)\right\} \xi_{0}^{2}, \\
n_{2} & \equiv 3\left\{2 s_{0}^{2} \xi_{0}^{2}+15\right\} t_{0}^{2}+\left\{2 s_{0}^{2} \xi_{0}^{4}-3\left(2 s_{0}^{2}+1\right) \xi_{0}^{2}-9\left(3 s_{0}^{2}+7\right)\right\} \xi_{0} t_{0}+ \\
& +\left\{3 s_{0}^{2} \xi_{0}^{2}+3\left(2 s_{0}^{4}+5 s_{0}^{2}+8\right)\right\} \xi_{0}^{2}, \\
n_{3} & \equiv 24 t_{0}^{3}+\left\{2 s_{0}^{2} \xi_{0}^{4}+\left(6 s_{0}^{2}-1\right) \xi_{0}^{2}-27\right\} \xi_{0} t_{0}^{2}+ \\
& +\left\{-7 s_{0}^{2} \xi_{0}^{2}+9\left(-3 s_{0}^{2}+1\right)\right\} \xi_{0}^{2} t_{0}+\left\{2 s_{0}^{4} \xi_{0}^{2}+3\left(2 s_{0}^{4}+5 s_{0}^{2}\right)\right\} \xi_{0}^{3}, \\
d & \equiv\left\{2 s_{0}^{2} \xi_{0}^{2}+3\right\} t_{0}^{2}-\left\{2 s_{0}^{2} \xi_{0}^{2}+\left(5 s_{0}^{2}+1\right)\right\} \xi_{0} t_{0}+\left\{2 s_{0}^{4}+s_{0}^{2}\right\} \xi_{0}^{2},
\end{aligned}
$$

with $s_{0} \equiv \operatorname{sech} \xi_{0}$ and $t_{0} \equiv \tanh \xi_{0}$.

The coefficients $\alpha, \delta$ and $\gamma$ are, thus, functions of $\kappa_{0} \equiv \omega_{0}^{2} h / g$. As $\kappa_{0} \rightarrow 0, \kappa_{0} \rightarrow \xi_{0}^{2}$ and $\varrho_{1} \rightarrow$ $-4 / 9, \varrho_{2} \rightarrow 1 / 63$ and $\varrho_{3} \rightarrow 1 / 945$, so that we recover the Padé [4/4] approximation Madsen and Schaffer (1998); Gobbi et al. (2000). In equations (11), there are four possible combinations depending on the signs, equivalent to the four possible solutions discussed by Madsen and Schaffer (1998).

Figure 4 shows the three functions $\alpha, \delta$ and $\gamma$ in all four cases. For " $+\&+$ " the values are similar to the values by M98 and G12 in Table 1. However, all four solutions give the same results in terms of linear dispersion.
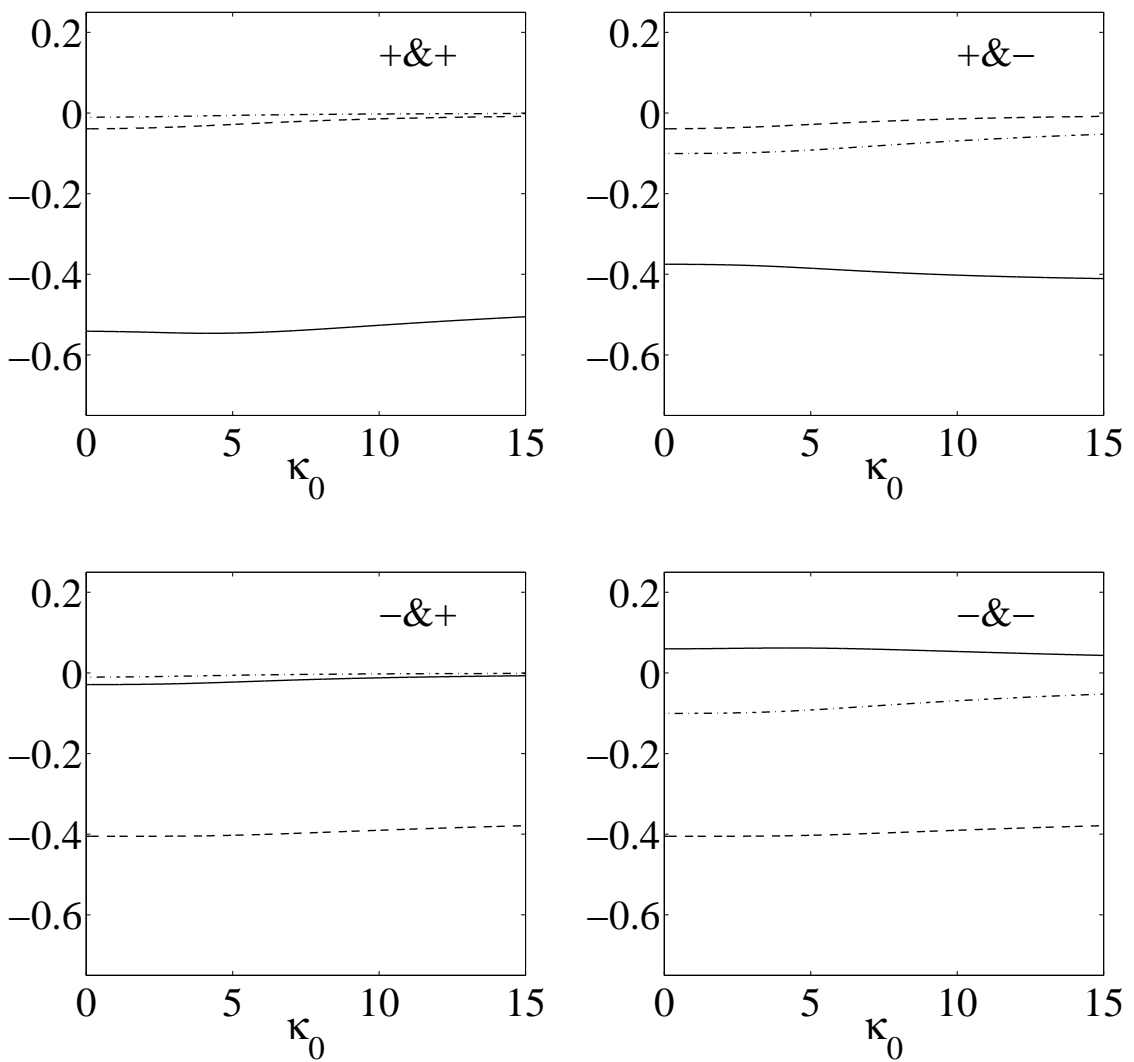

Figure 4: Coefficients $\alpha$ (full line), $\delta$ (dashed line), $\gamma$ (dash-dotted line), which are functions of $\kappa_{0} \equiv \omega_{0}^{2} h / g$, depending on the signs considered in equations (11). For instance, the case " $+\&-$ " results from considering " +" in equation (11a) and "_" in equation (11b). 